\title{
LAS COMPETENCIAS INTERCULTURALES EN LA SOCIEDAD DEL CONOCIMIENTO: REFLEXIONES Y ANÁLISIS PEDAGÓGICO
}

INTERCULTURAL COMPETENCE IN THE KNOWLEDGE SOCIETY: APPROACHES AND A PEDAGOGICAL ANALYSIS

\author{
Diana Priegue Caamaño; diana.priegue@usc.es \\ Juan J. Leiva Olivencia; juanleiva@uma.es \\ Universidad de Málaga
}

\section{RESUMEN}

El artículo pretende indagar en el potencial de la tecnología en la rentabilización del espacio digital de comunicación para el desarrollo de competencias de corte intercultural Entre las conclusiones destacan sobremanera dos cuestiones. De una parte, la necesidad de articular nuevas fórmulas que potencien el nivel de implicación de instancias y agentes educadores. De otra, la urgencia de apurar la preparación del profesorado en el aprovechamiento de la potencialidad pedagógica de los soportes digitales.

PALABRAS CLAVE: Sociedad del conocimiento, competencias interculturales, tecnología, digiculturalidad, comunidades de aprendizaje, aprendizaje cooperativo.

\section{ABSTRACT}

The paper is aimed at studying the potential of the technology in making the most of the digital communication space for the development of cross-cutting intercultural skills. . Among the conclusions drawn, two issues truly stand out. On the one hand, the need to create new approaches could enhance the level of involvement of education authority and agents. On the other hand, there is the need to rush the teachers' training in making the most of the educational potential of digital media.

KEY WORDS: Knowledge society, intercultural skills, technology, cyberculture, learning communities, cooperative learning. 


\section{INTRODUCCIÓN}

Los continuos avances tecnológicos y el mayor protagonismo que han adquirido flujos migratorios, plantean nuevas exigencias, demandas y desafíos al ámbito pedagógico. Nos encontramos inmersos en una sociedad postmoderna y multicultural que aspira a la construcción de la interculturalidad en lo social y presencial, y a la digiculturalidad en lo cultural y virtual (Leiva \& Moreno, 2011). Todo ello requiere que reflexionemos en mayor medida sobre el nuevo papel que, desde un paradigma inclusivo, compresivo e intercultural, han de asumir las instituciones educativas aprovechando las posibilidades y oportunidades que nos ofrecen las TIC para superar esa distancia entre la escuela y las realidades socioculturales. Atendiendo a lo expuesto por De Pablos (2009), al igual que el uso de las TIC en la enseñanza está teniendo un papel muy importante para lograr un replanteamiento metodológico, didáctico, curricular, organizativo en todos los niveles educativos con la mirada puesta en la optimización de los índices de calidad; también constituyen un instrumento valioso para aproximar culturas diferentes, reflexionar sobre la cultura de la diversidad, y promover una comunicación intercultural. Del mismo modo, para materializar los conceptos de interactividad, interconexión, instantaneidad, intercambio, conocimiento, enriquecimiento y ciudadanía intercultural, es necesaria una estrategia metodológica basada en el aprendizaje cooperativo, participativo y en red entre los centros de diversos lugares de nuestro mundo. Y para lograr este propósito las TIC constituyen una herramienta fundamental e imprescindible, no sólo por crear una comunidad donde los estudiantes pueden interactuar y compartir el conocimiento, sino por su potencial para transformar las prácticas educativas (Kozma y Anderson, 2002).

Es más, no hay duda que Internet y las redes sociales ponen a disposición de las escuelas inclusivas e interculturales todo lo necesario para generar y construir comunidades virtuales de aprendizaje intercultural, a través de multitud de herramientas susceptibles de favorecer la comunicación, la construcción compartida de conocimiento y el intercambio de información acerca de experiencias en educación intercultural entre todos los miembros de las comunidades educativas (De Haro, 2010).

\section{COMPETENCIAS INTERCULTURALES, TIC Y SOCIEDAD DEL CONOCIMIENTO}

Basándonos en el novedoso trabajo de Borrero y Yuste (2011), podemos afirmar que la incorporación de las TIC en los ámbitos educativos, tanto formales como no formales, ofrece múltiples y nuevas oportunidades de acceso a la información y gestión del conocimiento. Hoy en día los canales de comunicación se multiplican vertiginosamente, la tecnología no para de innovar y el tiempo dedicado al consumo de los medios de comunicación crece a un ritmo acelerado. Tal y como destaca Francisco (2010) lo importante no es adquirir o asimilar información, sino integrarla y reconstruirla críticamente en el propio -y personalconocimiento del saber, el saber en conocimiento y el conocimiento en cultura.

En este siglo XXI venimos observando que existe una preocupación social y cultural sobre el nivel de competencias que tienen los jóvenes en el uso de las TIC, que se ha visto reflejada en la literatura científica-pedagógica de los últimos años (De Pablos, 2009; Monereo, 2009; 
Prendes, Castañeda \& Gutiérrez, 2010) y ha traspasado los muros de la escuela para impregnar todos los ámbitos sociales y comunitarios. La digiculturalidad es una competencia digital de conocimiento y valoración de la diversidad cultural a través de las TIC y con unas características complejas en su desarrollo metodológico y conceptual. Como primera característica conceptual, destacamos la necesidad de multialfabetizar a nuestro alumnado para que sepa adaptarse a los diferentes cambios y necesidades de la sociedad de hoy. En este sentido, Area (2010) en Borrero y Yuste (2011) destaca las dimensiones o ámbitos de aprendizaje de la alfabetización tecnológica, que resumimos en el siguiente cuadro:

\begin{tabular}{|c|c|c|}
\hline Dimensión & Saber & Descripción \\
\hline Instrumental & Acceder a la información & $\begin{array}{l}\text { Adquirir las habilidades instrumentales } \\
\text { para la búsqueda de información y uso } \\
\text { de las tecnologías }\end{array}$ \\
\hline Cognitiva & $\begin{array}{l}\text { Transformar } \\
\text { información } \\
\text { conocimiento } \\
\end{array}$ & $\begin{array}{l}\text { Saber plantear problemas, analizar e } \\
\text { interpretar con significado la } \\
\text { información }\end{array}$ \\
\hline Sociocomunicacional & Expresarse y comunicarse & $\begin{array}{l}\text { Disponer de las habilidades para crear } \\
\text { documentos, difundirlos } \\
\text { interaccionar socialmente }\end{array}$ \\
\hline Axiológica & $\begin{array}{l}\text { Usar democrática y } \\
\text { éticamente la información }\end{array}$ & $\begin{array}{l}\text { Desarrollo de actitudes, valores y } \\
\text { prácticas sociales éticas }\end{array}$ \\
\hline
\end{tabular}

La segunda característica de la digiculturalidad corresponde a su desarrollo y aplicabilidad práctica en red. Esto significa que fundamentalmente tiene sentido pedagógico en tanto se genera y construye a partir de los valores de cooperación e interacción cultural democrática. En efecto, la digiculturalidad emerge con fuerza a través de plataformas y comunidades virtuales que son a la vez camino y destino de una conciencia comunitaria e intercultural donde lo importante es crecer individual y socialmente valorando positivamente la diversidad cultural y los valores universalmente aceptados contemplados en los derechos humanos (Martínez, 2010). Así mismo, no queremos terminar este apartado sin exponer qué es lo que las TIC están aportando al mundo de la interculturalidad. En este sentido nos gustaría destacar cuatro características básicas que servirán de soporte para el siguiente punto. Así, está claro que a través de las TIC podemos:

- Motivar a los más jóvenes a participar en la generación de comunidades virtuales.

- Promover el conocimiento y reconocimiento de las diferencias culturales como claves positivas de enriquecimiento personal y social.

- Facilitar la comunicación intercultural a través del contacto de personas y culturas muy diversas.

- Favorecer la participación activa y colaborativa gracias al empleo sistemático de herramientas virtuales de la Web 2.0.

Por tanto, el desafío que plantea la generación de todos los niveles educativos, especialmente en aquel alumnado universitario de las titulaciones conducentes a ser profesionales de la educación sería adquirir competencias interculturales desde un nuevo enfoque de formación universitaria, más preocupada por los principios de procedimiento didáctico-estratégicos y los valores educativos de la diversidad y el respeto cultural, que por meras aplicaciones o conocimientos teóricos de una aparente y aséptica tecnología educativa. Esto, de acuerdo con lo planteado por Valls \& Otros (2002), supone que el 
profesorado universitario comience a incentivar y a generar en las aulas universitarias procesos didácticos en términos de comunidades de aprendizaje, lo cual exige la puesta en marcha de todo un conjunto de iniciativas que, desde la transformación de la organización escolar y de su funcionamiento, culmine en la acogida y promoción de la diversidad cultural como un eje formativo fundamental en el aprendizaje de las competencias digiculturales. A examinar esta cuestión dedicaremos las páginas siguientes.

\section{EL DESARROLLO DE COMPETENCIAS INTERCULTURALES A TRAVÉS DE LA COOPERACIÓN EN LA RED}

Sin lugar a dudas, que Internet haya pasado de ser un espacio de lectura a convertirse en un espacio de lectura-escritura ha sido el motor de una nueva revolución tecnológica: el auge de la web 2.0. A todas luces es obvio que lo realmente relevante de las TIC no es su potencial para facilitar el acceso a la información sino sus posibilidades para incrementar los procesos de comunicación interpersonal al margen de las dimensiones espacio-tiempo y la construcción compartida del conocimiento. Es lo que Tubela \& Vilaseca (2005) definen como modelo de ciberespacio de comunidad, cuyo eje central es la comunicación entre usuarios, el establecimiento de vínculos entre los participantes y la gestión democrática del conocimiento que se construye en la comunidad. Así pues, las comunidades virtuales de aprendizaje se caracterizan porque, además de constituirse como una comunidad de intereses o de participación, utilizan los soportes digitales como infraestructura para consolidar y ampliar las redes de comunicación y de intercambio, al tiempo que como instrumento para promover el aprendizaje de sus miembros (Coll, Bustos \& Engel, 2007). Y al respecto no podemos olvidar que, si la educación es un proceso de comunicación, cuando hablamos de interculturalidad esta afirmación cobra aún mayor firmeza por ser este el ámbito donde el sujeto de la educación no puede entenderse aisladamente (Santos, Lorenzo $\&$ Priegue, 2011). Seguidamente se recogen las etapas que, a nuestro parecer, conformarían la creación de una comunidad virtual de aprendizaje. Para ello hemos realizado una adaptación de la propuesta de Salmon (2000; cit en Martín \& Quiroz, 2006, 312):

- 1a etapa: Acceso y motivación. En esta primera fase el principal objetivo es reconocer la utilidad práctica que una comunidad virtual de aprendizaje puede proporcionar al grupo en general y a cada persona de manera individual.

- 2a etapa: Compromiso y socialización en línea. Se trata, principalmente, de establecer identidades virtuales y vínculos entre los participantes. La calidad de las relaciones establecidas determinará en gran medida el desarrollo de las etapas posteriores.

- 3a etapa: Intercambio de información. La finalidad es conseguir compartir información relevante en función de las propias necesidades, las de los otros participantes y de la comunidad en su totalidad. Además, en esta etapa es importante que el alumnado perciba los aspectos positivos derivados del proceso.

- 4a etapa: Construcción del conocimiento. Ésta es una de las fases clave pues el objetivo es lograr la construcción co-participativa de nuevos conocimientos a partir de las aportaciones de todos los participantes. 
- 5a etapa: Desarrollo. Se trata de usar el proceso para alcanzar metas compartidas y objetivos individuales. Es decir, llegados a este punto, los participantes deberían poder integrar los conocimientos derivados de la implicación en la red con lo aprendido en otros contextos.

- 6a Consolidación. El propósito de esta última etapa es que la red permanezca en el tiempo o sea el origen de nuevas redes con objetivos más amplios. Para que podamos hablar de consolidación el nivel de implicación de los participantes debe mantenerse, lo que a su vez exige disponer de un elevado grado de motivación.

A modo de síntesis podríamos decir que las claves que favorecen el éxito de este tipo de comunidades pasan por compartir objetivos o intereses comunes, tener un sentimiento de pertenencia al grupo, y un compromiso activo de todos los miembros. Por otra parte, tampoco debemos pasar por alto que los estudios realizados a nivel internacional han demostrado que la participación del alumnado en estas estructuras incide positivamente en el rendimiento académico y en la motivación de los estudiantes (Thomas, 2000). A todo ello debemos añadir su gran potencial para la creación de espacios de interacción cooperativa. Naturalmente, de las potenciales virtualidades que presentan las técnicas de aprendizaje cooperativo nos interesa especialmente la mejora que parecen producir en el esfuerzo por el aprendizaje entre compañeros, porque aumentan la motivación general, la implicación en las tareas y la ayuda que se proporcionan entre sí, amén de que propicia la reducción de prejuicios étnicos (Slavin, 1999; Santos, Lorenzo \& Priegue, 2009). Concretamente, el potencial de estos soportes en el desarrollo de competencias de corte intercultural tiene que ver con cuatro aspectos fundamentales en los que nos centraremos a continuación.

En primer lugar, todos los participantes aportan su actividad a fin de alcanzar una meta común, siendo ésta otra de las premisas básicas del aprendizaje cooperativo. Pensemos en la idoneidad de estas estructuras para la asunción de responsabilidades compartidas y el establecimiento de metas de equipo, por encima de comportamientos individualistas y de la competitividad, relegando a un segundo plano las diferencias que puedan existir entre los participantes y fortaleciendo el sentimiento de comunidad y necesidades compartidas. Así pues, una de las claves del éxito reside en crear interdependencia positiva entre los integrantes del grupo, es decir, la necesidad de que trabajen juntos para conseguir los objetivos establecidos en y para la comunidad.

Otro de sus aspectos positivos tiene que ver con su contribución a la educación en y para la democracia. La naturaleza del uso y funcionamiento de estos soportes favorece el establecimiento de estructuras de participación democráticas donde se combinan aprendizajes de tipo cognitivo, social y emocional. El diálogo, la confrontación de ideas y la participación son herramientas esenciales para el buen funcionamiento de estas estructuras $y$, al mismo tiempo, todos ellos son elementos fundamentales para el desarrollo de una sociedad democrática (Santos y Lorenzo, 2009).

El tercer aspecto a destacar es que la desaparición del espacio, como distancia física entre los interlocutores, no lleva emparejada la desaparición del entorno social que rodea a esos mismos interlocutores (Martínez \& Prendes, 2003). Es evidente que los procesos de comunicación se establecen y se construyen desde los espacios de significación cultural de cada individuo, por lo cual la implicación en redes de este tipo posibilita el conocimiento de realidades y representaciones culturales diferentes, lo que es susceptible de contribuir a la 
crítica de las propias identidades culturales (individuales y colectivas), así como a la generación de una cultura de la diversidad en el espacio virtual.

En último lugar, la participación en estas redes favorece la aceptación y la valoración positiva de la diversidad. No nos referimos únicamente a las diferencias que puedan existir por razones culturales, o de género, sino a la diversidad intrínseca que nos es propia como seres particulares.

Una vez expuestos los aspectos fundamentales de nuestra propuesta hemos de resaltar que existen varios entornos virtuales que hacen posible su puesta en práctica en las aulas de todos los niveles educativos. Además de la plataforma Moodle, disponemos de otros sistemas menos conocidos, caso del BSCW (Basic Support for Cooperative Working) que, como su propio nombre indica, constituye un entorno basado en el trabajo cooperativo en la red que, además de la navegación y el acceso a la información, permite compartir y construir documentos a través de distintas plataformas (Windows, Macintosh o Unix) y establecer una agenda común entre los miembros del grupo. Dispone, además, de distintas herramientas para supervisar la creación, lectura o modificación de información a través de un sistema de alerta que nos informa de la actividad de otros usuarios del entorno. Todo ello sin que sea necesario instalar ningún software adicional. Aunque en este trabajo tan sólo nos refiramos a una de ellas, en los últimos años se han desarrollado numerosas herramientas groupware bajo licencias de software libre de fácil acceso, con prestaciones muy similares a las que nos proporcionan distintas marcas comerciales, habitualmente menos accesibles por su elevado coste. Ahora bien, sabiendo que disponemos de los soportes tecnológicos necesarios para desarrollar un planteamiento como el que desde aquí proponemos, probablemente su rentabilización desde un punto de vista intercultural dependerá en gran medida de la formación y actitudes no sólo de los docentes, sino también de la comunidad escolar en su conjunto, además de la formación intercultural del profesorado en iniciativas y diseños didácticos especializados en el empleo reflexivo y crítico de las TIC para la promoción de las relaciones interculturales entre los diferentes agentes educativos (Luchtenberg, 1995; Tondeur, Devos y Van Houtte, 2009).

\section{EXPERIENCIAS Y PROPUESTAS: DE LA INTERCULTURALIDAD A LA DIGICULTURALIDAD}

En las aulas en mayor o menor grado y gracias al buen quehacer profesional de los docentes se han ido integrando las TIC en sus programaciones y procesos de enseñanza-aprendizaje acordes con su aparición y contexto (televisión, vídeo, retroproyectores, internet, redes sociales,...). Además, al margen de las carencias que el profesorado percibe en su formación para el manejo y aprovechamiento de las TIC, los estudios dan cuenta de la mayor sensibilidad que ha ido desarrollando este colectivo hacia la necesidad de introducir las tecnologías en el proceso de enseñanza-aprendizaje, sobre todo, por su gran potencial para diversificar el proceso de enseñanza-aprendizaje y de atender, en mayor medida, las necesidades particulares que pueda presentar el alumnado, especialmente, el procedente de la inmigración.

No podemos continuar sin reconocer el notable esfuerzo económico que ha realizado la administración educativa para proporcionar a los centros escolares el equipamiento tecnológico que exige una sociedad que apuesta por la mayor rentabilización del espacio 
digital. A este nivel uno de los referentes en nuestro país lo encontramos en la Comunidad Autónoma de Extremadura donde en el año 1999 se presentaba el "Plan Estratégico para el Desarrollo de la Sociedad de la Información", siendo el sector educativo uno de los pilares sobre los que se asentaba dicho desarrollo. Tal y como plantean Borrero y Yuste (2011), es a partir de este momento cuando en Extremadura aparece lo que se ha denominado "Las TIC en el Aula". En este contexto nace LinEx que no sólo es un sistema operativo sino que actualmente posee un conjunto enorme de importantes y variadas aplicaciones informáticas que cubre las necesidades de usuarios básicos y avanzados al tiempo que ofrece diversidad de herramientas para uso en el aula. A partir del año 2000 se inicia el despliegue de la Red Tecnológica Educativa, que se completa en el año 2003. Las TIC se hacen presentes en el aula, no son ya un recurso externo sino una herramienta más, como la tiza o la pizarra. Todos los centros educativos públicos, disponen de conectividad de banda ancha con un parque informático de unos 70.000 ordenadores, lo que posibilita una ratio de un ordenador por cada dos alumnos en secundaria y uno por cada seis en primaria: aparece el concepto de "Aula Tecnológica".

Ahora bien, la educación intercultural precisa de objetivos más amplios: reclama instituciones y personas que necesariamente trabajen en red, en comunidades de aprendizaje que promuevan la diversidad cultural como una riqueza y no una lacra perturbadora de la convivencia o del rendimiento académico, lo cual supone todo un conjunto de iniciativas que deberán culminar con la transformación de la organización escolar (Leiva y Moreno, 2011). En efecto, lo que necesitamos, es impulsar procesos de comunicación e intercambio intercultural intra e intercentros fundamentados en el reconocimiento del potencial educativo de la diversidad y de la acción comunitaria empleando nuevos entornos virtuales de comunicación e interacción.

De hecho, desde el Observatorio Escuela 2.0, del Ministerio de Educación (http://recursostic.educacion.es/observatorio/web/es/equipamiento-tecnologico/aulas-

digitales/784-recursos-web-para-la-atencion-a-alumnado-inmigrante-en-centros-de-es), se plantea que ante el fenómeno de la inmigración y la diversidad cultural, las instituciones escolares deben tener presente en su gestión que en ellas se encuentra alumnado procedente de diferentes culturas y con diferentes lenguas, que tienen que integrarse en un marco de convivencia común y experimentar la diversidad no como un obstáculo, sino como un fenómeno positivo; comprometerse con el principio de igualdad de oportunidades en la educación y la convivencia y vertebrarse desde un enfoque comunitario del hecho educativo y de la construcción de una nueva ciudadanía, necesariamente intercultural. Estamos ante un proceso que debe ser liderado por las instituciones educativas y sus profesionales. Sin embargo, al igual que ocurre con otras cuestiones de gran relevancia pedagógica, la escuela y los docentes no pueden asumir en solitario la mejor formación del alumnado, sobre todo, cuando sabemos que lo que precisamos es impulsar procesos formativos fundamentados en los principios de la interculturalidad y, en consecuencia, debemos contar con el compromiso de las demás agencias educadoras. En este sentido, resulta muy relevante destacar el trabajo realizado en la Comunidad Autónoma de Andalucía, donde la Consejería de Educación viene impulsando el desarrollo de nuevas estrategias educativas de cooperación, formación y participación de las familias. De hecho, la Consejería de Educación, a través de la Plataforma Educativa Helvia (accesible desde el siguiente enlace, http://www.juntadeandalucia.es/averroes/helvia/sitio/index.cgi?wid seccion=2\&wid item= 24), está facilitando la creación de redes de innovación e investigación, la base andaluza de recursos digitales (BARTIC), un banco de materiales digitales accesible a toda la ciudadanía 
donde se podrán buscar y descargar juegos, experiencias educativas, contenidos escolares y objetos de aprendizajes, así como espacios virtuales destinados a las comunidades educativas para facilitar la posibilidad a docentes, familias y alumnado de crear sus propias weblogs, foros y bitácoras en los que intercambiar reflexiones, ideas, propuestas y recursos educativos diversos.

De similar envergadura es el esfuerzo realizado en la Comunidad Autónoma de Galicia para poner en marcha desde el curso 2010-11 el "Proyecto Abalar" (http://www.edu.xunta.es/web/abalar), una iniciativa que tiene por objetivo potenciar la integración plena de las TIC en la práctica educativa en esta Comunidad y que apuesta por el concepto de centro educativo digital. Por y para ello, desde la Consellería de Educación e Ordenación Universitaria de la Xunta de Galicia se ha realizado una gran inversión económica a fin de dotar a los centros escolares de los recursos humanos y pedagógicos, así como de las infraestructuras y servicios necesarios, para el aprovechamiento cotidiano de la tecnología por parte de todos los agentes de la comunidad educativa. Además de promover distintas acciones formativas para mejorar la preparación del profesorado en el manejo y aprovechamiento de los soportes tecnológicos, en el marco del proyecto se ha creado un portal digital (Espazo Abalar) que aloja recursos educativos en soporte digital para los distintos niveles de enseñanza que los docentes pueden utilizar en las sus materias y dispone de varias aplicaciones telemáticas que facilitan la comunicación entre los distintos miembros de la comunidad educativa. Sin dejar de reconocer los avances que pueden derivarse de la puesta en marcha de soportes digitales que promueven la colaboración entre la escuela y los demás agentes educativos, probablemente el aprovechamiento de sus ventajas desde una perspectiva intercultural dependerá tanto de la buena voluntad de los docentes como de la formación que hayan recibido para ello. En este sentido, uno de los proyectos donde se puede observar con nitidez la vinculación y traducción práctica de la interculturalidad a través de las TIC es el proyecto E-Culturas (http://www.e-culturas.org), proyecto desarrollado por el Grupo IDEO, de la Universidad de Jaén, y que tenía como objetivo fundamental el establecer una plataforma virtual que sirviera especialmente al alumnado de educación primaria a mejorar su capacidad de intercambio y comunicación cultural con alumnado perteneciente a centros de diversos países latinoamericanos. Apuntando en esta misma dirección tampoco podemos olvidar los programas educativos de la Unión Europea que promueven el intercambio intercultural y la alfabetización digital. De hecho, tal y como plantea Martínez (2010), el objetivo fundamental de estos programas formativos es reforzar la cohesión social, el desarrollo personal, el diálogo intercultural y la participación activa de los jóvenes ciudadanos europeos. Una de las iniciativas más conocidas es el eTwinning (http://www.etwinning.net/es/pub/index.htm), que permite la comunicación e intercambio de experiencias educativas interculturales entre escuelas distantes geográfica y culturalmente.

Por otro lado, también mencionamos otras experiencias de educación inclusiva e intercultural a través de las TIC, en este caso, en la Comunidad Foral de Navarra donde en los últimos años se está promoviendo el desarrollo de la interculturalidad a través de las TIC. En este sentido, el Departamento de Educación del Gobierno de Navarra ha convocado resoluciones de financiación o de subvención a proyectos educativos y experiencias de Educación Intercultural, desarrollados en centros de enseñanza, públicos y concertados, de la Comunidad Foral de Navarra. Los proyectos son impulsados por equipos de trabajo formados por profesorado de los centros, y donde pueden colaborar ONGs, Servicios Sociales, Ayuntamientos, otras entidades y miembros de comunidades. Algunos ejemplo de 
los 17 proyectos desarrollados durante el curso 2010/2011, son los siguientes: "Diferentes pero iguales en derechos" del IES de Berriozar (www.navarra.es/NR/...FD49.../PROYECTOSmulticulturalidad.doc). Llevado a cabo en todos los niveles y área, participa el colectivo social "Entreculturas", y los objetivos fundamentales son: fomentar las relaciones y la empatía en el alumnado; conocimiento de las diferentes culturas y religiones e implicación especial de los delegados de clase en la organización de actividades de educación intercultural. Éstas conducen a la reflexión y la concienciación a través de relatos, juegos, vídeos y jornadas interculturales La evaluación se efectuará mediante encuestas, entrevistas personales a una muestra del alumnado y del profesorado implicado. También, nos encontramos con el proyecto titulado "Corresponsales sin fronteras" del CEIP Teresa Bertrán de Lis, de Caderita (www.educacion.navarra.es/portal/digitalAssets/.../41576 ProyecCas.doc). En este caso, se trata de un proyecto a realizar a través de los medios de información y comunicación (TIC) para el desarrollo de habilidades sociales e interculturales. Estas se introducen por medio de cuentos y juegos del mundo. En la etapa de educación Infantil se desarrollan cuentos y juegos del mundo así como la elaboración de una sección del periódico escolar y actividades en torno a la televisión, mientras que en Educación Primaria se llevan a cabo actividades tales como murales, programas de radio y T.V., relatos de cuentos del mundo, juegos de otras culturas, exposiciones. Todo ello a partir de una plataforma virtual donde existen numerosos recursos didácticos a disposición de la comunidad educativa. Precisamente, en la Web de Aula Intercultural, del sindicato FETE-UGT, se encuentra todo un apartado dedicado a experiencias dedicadas a la promoción de la educación intercultural a través de las TIC (http://www.aulaintercultural.org/experiencias/).

Ciertamente, son ya varios los centros educativos los que vienen desarrollando proyectos educativos específicos para impulsar y generar interculturalidad a través de las redes sociales y plataformas virtuales en dominios libres. A modo de ejemplo podemos referirnos al esfuerzo realizado desde los grupos de trabajo organizados en los IES Victoria Kent y Dunas de las Chapas en la provincia de Málaga durante los últimos cursos escolares $2009 / 2010$ y $2010 / 2011$. Aunque probablemente existan experiencias de corte similar a las que podríamos hacer referencia, la escasa de difusión de muchas de las iniciativas llevadas a cabo en los centros educativos limita sus posibilidades de desarrollo en otros contextos. No se trata sólo de un proceso de transformación tecnológico o de incorporación acrítica de un nuevo espacio de encuentro cultural, sino que el hecho de participar de un proceso de toma de decisiones contribuye a la mejor formación en valores democráticos tanto para el alumnado como para el profesorado, y, por supuesto, para las familias y el resto de agentes de la comunidad educativa. Hablamos, en definitiva, de vivir la escuela y la interculturalidad como una construcción comunitaria participativa (Puigvert \& Otros, 2006). Teniendo esto presente, lo que ahora exponemos son algunos de los objetivos más relevantes en relación al aprovechamiento de las TIC y el desarrollo de estrategias prácticas que, desde los principios de la interculturalidad, favorezcan una mayor implicación de la comunidad educativa en los procesos educativos:

- Potenciar la comunicación con la comunidad educativa y su entorno social: hojas informativas, páginas Web, correo electrónico, en el que se divulgue el Centro y su oferta educativa, relación con las AMPAs, e intercambio de información con las familias. 
- Fomentar actividades de participación virtual de toda la comunidad educativa, a través de la puesta en práctica de un periódico digital, blogs por cursos o niveles educativos, exposición de webquest por parte de los docentes, foros y debates a través de la plataforma virtual del centro escolar, planteando encuestas, jornadas virtuales de puertas abiertas.

- Incrementar la utilización de las TIC por parte de toda la comunidad educativa, especialmente de las familias, con el fin de promover su participación activa en la elaboración de los documentos pedagógicos del Centro, potenciando la Wiki y el Foro, así como el correo electrónico como medio de aportar recomendaciones y aportaciones comunitarias a la vida educativa del centro escolar, así como para contactar con el profesorado.

Hemos depositado una gran confianza en la institución escolar y en sus profesionales pero a todas luces es obvio que las acciones puestas en marcha alcanzarán más y mejores resultados si contamos el apoyo y el compromiso de la comunidad educativa en su conjunto. La gestión colectiva de un desafío que afecta a la sociedad civil en su conjunto posibilitará la puesta en práctica de los principios de una ciudadanía democrática donde, como es bien sabido, la participación y la asunción compartida de las responsabilidades ocupan un lugar central.

\section{CONCLUSIONES FINALES}

Los numerosos cambios que se han producido en un breve periodo de tiempo y, sobre todo, la velocidad con la que han tenido lugar dichas transformaciones, han sido el origen de una preocupación creciente en torno a la capacidad de la escuela para formar a la ciudadanía del siglo XXI. Y aunque resulta harto complicado establecer prioridades cuando abordamos un asunto de relevancia incalculable como es la educación de los jóvenes, uno de los retos más importantes de nuestro tiempo tiene que ver con el desarrollo de aquellas competencias que, desde el respeto y valoración de una sociedad positivamente multicultural e interconectada, facilitan tanto el conocimiento de otras perspectivas y percepciones sobre la realidad como el análisis crítico de las propias. Ciertamente, sería una falacia negar que en los últimos años se han producido numerosos avances, pero no es menos cierto que en el seno de nuestras escuelas persisten problemáticas de las que ya hemos dado cuenta en otras ocasiones, caso de la necesidad de intensificar las relaciones entre familias, escuela y comunidad, asunto ampliamente demostrado que no precisa que añadamos más o mejores argumentos. Pero en lo que sí hemos de insistir es en la búsqueda de nuevas fórmulas susceptibles de optimizar el nivel de implicación y compromiso de instancias y agentes educadores. Precisamente por ello creemos que una de las claves se sitúa en la mayor rentabilización del espacio digital de comunicación para fortalecer una conectividad de corte intercultural mediante la configuración de nuevos espacios de colaboración y aprendizaje que favorecen el desarrollo de estructuras de trabajo cooperativo en la red. Esto supondrá mejoras en el conocimiento, la comprensión y la convivencia intercultural en los más jóvenes, consumidores y también creadores de significados culturales cada vez más diversos, cambiantes y dinámicos.

Ahora bien, antes de terminar quizás debamos preguntarnos si la buena voluntad de los docentes va a ser suficiente para aprovechar las ventajas que pueden introducir los soportes digitales en los procesos de enseñanza-aprendizaje, o bien si otra de las prioridades se sitúa 
en el ajuste de la preparación del profesorado a los requerimientos de la sociedad actual, entre ellos, la mejor capacitación para gestionar de manera eficaz las aulas con apreciable heterogeneidad étnico-cultural. Así mismo, el desarrollo de las competencias interculturales en la sociedad del conocimiento implica que los docentes deben dinamizar propuestas que construyan comunidades de aprendizaje, luego la formación y la comunicación debe ir adquiriendo un matiz comunitario básico en la configuración de redes educativas dinámicas donde familias, profesorado y alumnado tengan mejores y mayores oportunidades de conocimiento y comprensión intercultural.

\section{REFERENCIAS}

AREA, M. (2010). Enseñar la competencia digital e informacional [diapositiva]. Peñaranda de Bracamonte, $27 . \quad$ (http://www.slideshare.net/citafgsr/manuel-areacita2010?from=share email logout2) (17/08/2011)

BORRERO, R. y YUSTE, R. (2011). "Digiculturalidad.com. Interculturalidad y TIC unidas en el desarrollo del enfoque competencial del curriculum", en LEIVA, J. y BORRERO, R. (Coords.). Interculturalidad y escuela. Perspectivas pedagógicas en la construcción comunitaria de la escuela intercultural (pp.145-164), Barcelona: Octaedro.

COLL, C., BUSTOS, A. \& ENGEL, A. (2007). Comunidades de práctica complementarias: el equipo del programa y la comunidad en línea del curso. Revista Electrónica de la Educación: Educación y Cultura en la Sociedad de la Información, 8(3) (www.usal.es/ teoriaeducacion) (20/09/2011).

CUMMINS, J. \& SAYERS, D. (1995). Brave new schools: Challenging cultural illiteracy through global learning networks. New York: St. Martins Press

DE HARO, J.J. (2010). Redes Sociales para la educación. Madrid: Anaya.

DE PABLOS, J. (Coord.) (2009). Tecnología Educativa. Málaga: Aljibe

FRANCISCO, A. (2010). Construyendo ciudadanía participativa en la sociedad de la información. Pixel Bit, 37; 135-146.

KOZMA, R.B., \& ANDERSON, R.E. (2002). Qualitative case studies of innovative pedagogical practices using ICT. Journal of Computer Assisted Learning, 18, 387-394.

LEIVA, J. \& MORENO, N. (2011). Construyendo comunidades virtuales de aprendizaje intercultural en la escuela. Actas del VI Encuentro "Tendiendo puentes hacia la interculturalidad". Málaga: Facultad de Ciencias del Trabajo de la Universidad de Málaga.

LUCHTENBERG, S. (1995). Intercultural Communicative Competence: a challenge in multicultural and anti-racist education, European Journal for Intercultural Studies, 6 (2), 1223.

MARTíN, M.T. \& QUIROZ, C. (2006). Perfil y funciones del educador social, en López-Barajas, E. (Coord.). Estrategias de Formación en el Siglo XXI. Barcelona: Ariel; 285-316.

MARTínEZ, F. (2010). Las redes digitales como marco para la multiculturalidad. Alcalá de Guadaíra (Sevilla): MAD.

MARTíNEZ, F. \& PRENDES, M.P. (2003). ¿A dónde va la educación en un mundo de tecnologías?, en MARTínEZ, F. (Comp.). Redes de comunicación en la enseñanza. Las nuevas perspectivas del trabajo corporativo. Barcelona: Paidós; 281-300. 
MONEREO, C. (2009). Internet, un espacio para desarrollar las competencias básicas, en MONEREO, C. (Coord.), Internet y competencias básicas. Aprender a colaborar, a comunicarse, a participar, a aprender. Barcelona: Graó; 5-26.

PRENDES, M.P; CASTAÑEDA, L. \& GUTIÉRREZ, I. (2010). Competencias para el uso de TIC de los futuros maestro. Comunicar, 35; 175-182.

PUIGVERT, L. \& OTROS (2006). Teorías y Sociedades Dialógicas. Nuevas transferencias ciencia y sociedad en la era del conocimiento. Madrid: Ministerio de Ciencia y Tecnología.

SANTOS, M.A. \& LORENZO, Mạ. (2009). A educación para a cidadanía e os profesores. Visión e desafío. Vigo: Xerais.

SANTOS, M.A., LORENZO, Mạ. \& PRIEGUE, D. (2009). Aprendizaje cooperativo: práctica pedagógica para el desarrollo escolar y cultural. Magis, Revista Internacional de Investigación en Educación, 1(2); 289-304.

_ (2011). (Red) Conectando a los profesores para el desarrollo de la interculturalidad. Revista Educación XX1 (en prensa).

SLAVIN, R.E. (1999). Aprendizaje cooperativo. Teoría, investigación y práctica. Buenos Aires: Aique.

THOMAS, J.W. (2000). A review of research on project-based learning. San Rafael, CA: Autodesk Foundation.

TONDEUR, J., DEVOS, G. y VAN HOUTTE, M. (2009). Understanding structural and cultural school characteristics in relation to educational change: the case of ICT integration, Educational Studies, 35 (2), 223-235.

TUBELA, I. \& VILASECA J. (2005). Sociedad del conocimiento, cómo cambia el mundo ante nuestros ojos. Barcelona: UOC.

VALLS, R. \& Otros (2002). Comunidades de aprendizaje. Transformar la educación. Barcelona: Graó.

Para citar este artículo:

PRIEGUE, D. \& LEIVA, J. J. (2012). Las competencias interculturales en la sociedad del conocimiento: reflexiones y análisis pedagógico. EDUTEC, Revista Electrónica de Tecnología Educativa, 40 Recuperado el dd/mm/aa de http://edutec.rediris.es/Revelec2/Revelec40/competencias interculturales sociedad conoc imiento reflexiones analisis pedagogico.html 